Volume 8. No. 10, October 2020

International Journal of Emerging Trends in Engineering Research

Available Online at http://www.warse.org/IJETER/static/pdf/file/ijeter218102020.pdf

https://doi.org/10.30534/ijeter/2020/218102020

\title{
Detection of Abnormalities in Kidneys Using Image Processing
}

\author{
P. Vijayakumar ${ }^{1}$, Prithiviraj Rajalingam ${ }^{2}$, R. Nandakumar ${ }^{3}$,S. Praveenkumar ${ }^{4}$, A. Joshua Jafferson ${ }^{5}$ \\ ${ }^{, 1}$ Associate professor, SRM Institute of Science \& Technology, Kattankulathur, Kancheepuram, Tamilnadu, India, \\ ${ }^{3}$ Professor, K S R Institute for Engineering and Technology, Tiruchengode \\ ${ }^{2,4,5}$ Assistant professor, SRM Institute of Science \& Technology, Kattankulathur, Kancheepuram, Tamilnadu, India
}

\begin{abstract}
This paper proposes a method to process advanced medical image models that provide the automation of kidneys abnormal deetction. In this paper, it has been designed in a way to identify the organ structures based on their features and look for abnormalities using erode and corrode image filling. The proposed method also invokes the segmentation algorithm on rendering filtering process for removal of noise resulting in recognition of image, and then connectivity analysis is performed, which differentiates the relation between neighborhood pixels that is used to detect boundaries. And based on the analysis and classification, we can calculate the desired probabilistic estimations of relevant images. The provoked method enables the increase in automation and higher accuracy of organs from the input stage of MRI datasets. The proposed method is evaluated with the available dataset and it shows a vast improvement in the performance classification.
\end{abstract}

Key words : Image acquisition, Enhancement, Parameter analysis, Connectivity analysis, Segmentation algorithms, Detection of tumors.

\section{INTRODUCTION}

Developing a system that supports increased automation of kidney detection [1] as well as their abnormalities (tumors, stones, cysts), which is capable of detecting and annotate the RoI [2] and also to annotate the organs and their potential abnormalities in a specific MRI dataset. Here it uses region-oriented segmentation to recognize the image of organs. The histogram values are normalized and the threshold value is fixed. This is followed by segmentation and labeling of the segmented images. Then connectivity analysis is performed to detect boundaries and based on the result, the exact problem can be identified. Image segmentation has been used to find the boundaries or edges between the tissues to find a size measurement and, by the image size declare the abnormalities.

Even though the ultrasound image is very easy and transferable and very protective but due to a large number of noises. It will affect the image. Speckle noise is one of the major noises which affect image processing. Speckle noise will reduce contrast and resolution. It leads to difficulty in requirement of the automatic segmentation process gets very high.

The segmentation of the kidney and finding the abnormalities can be used in surgical places for the operation called punctures. There are many advancements in computer-aided diagnosis system for the diagnosis of a disease. In this paper, the proposed method invokes the segmentation algorithm on rendering filtering process for removal of noise, resulting in recognition of image, and then connectivity analysis is performed, which differentiates the relation between neighborhood pixels that is used to detect boundaries.

\subsection{Problem Statement}

A Kidney is a very important organ in the human system. Kidney malfunctioning will threaten human life. It leads to the finding of kidney stone in human life at an early stage is a much more important factor. To remove the kidney stone from the human body, the most important process is to the position the kidney stone in the human body. In the ultrasound image, there will be more speckle noise and the image are in low contrast. Due to the speckle noise, the contrast and resolution of the image will get affected. It leads to the difficulty in finding the boundary of an image. In the ultrasound image, the boundary resolution is very low. This leads to the find the abnormality of small stones in the kidney for doctors. To address this issue, the modified image segmentation algorithm has been proposed.

\section{BLOCK DIAGRAM}

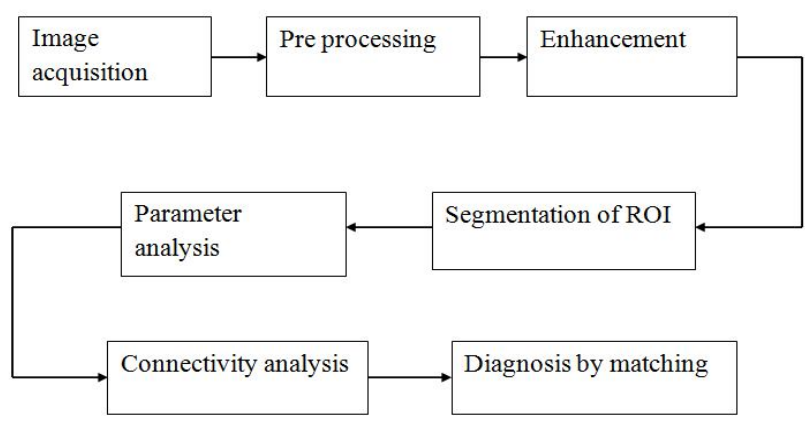

Figure 1: Schematic procedure for the detection of abnormalities. 
P. Vijayakumar et al., International Journal of Emerging Trends in Engineering Research, 8(10), October 2020, 6750 - 6756

Figure 1 shows the flow of abnormalities detection.

\subsection{Image Acquisition}

A camera captures the image, and it is converted into a right quality image to detect the abnormalities. This conversion process is called an Image Acquisition. Three steps do the process of image acquisition; the first process is the amount of energy reflected from the primary source (object). The second process is the optical device, which gives the energy to the source, and the last method is a quality of sensor which measures the amount of energy to be dissipated.

\subsection{Pre-processing}

Pre-processing is the second step after the image acquisition. Pre-processing actions are directed to remove or reduce the low-frequency background noise, masking the unwanted part in the image, and normalizing the image's intensity.[3] The pre-processing must be done before the computational process. [4]. The pre-processing is the process which will remove the noise and low contrast. The pre-processing will enhance the image quality and increase in contrast. The image will be smoothened and sharpened with better quality.

\subsection{Image Quality}

The next step after the pre-processing is the enhancement of image quality. By improving the image quality, the more noise in the image will be suppressed. Two processes will be followed to make an image look better quality. They are enhancement and restoration. The enhancement method uses the human visual response to improve the image quality. The restoration method is to add distortion to the image and reverse the degradation. [5].

\subsection{Median Filter}

The median filter replaces the median of the grey value in an image with a pixel's original grey level in a specific neighborhood. [6]. Figure 2 shows the operation to find the median value.

5-neighbothood
\begin{tabular}{|l|l|l|l|l|}
\hline $\mathbf{1 0}$ & $\mathbf{1 2}$ & $\mathbf{1 2}$ & $\mathbf{1 0}$ & $\mathbf{1 0}$ \\
\hline $\mathbf{1 0}$ & $\mathbf{1 0}$ & $\mathbf{1 2}$ & $\mathbf{3 2}$ & $\mathbf{3 2}$ \\
\hline $\mathbf{2 5}$ & $\mathbf{1 0}$ & $\mathbf{2 8}$ & $\mathbf{1 8}$ & $\mathbf{1 8}$ \\
\hline $\mathbf{2 5}$ & $\mathbf{2 5}$ & $\mathbf{3 2}$ & $\mathbf{3 2}$ & $\mathbf{1 8}$ \\
\hline $\mathbf{3 2}$ & $\mathbf{3 2}$ & $\mathbf{3 2}$ & $\mathbf{2 5}$ & $\mathbf{2 5}$ \\
\hline
\end{tabular}

Figure 2: Calculating the median value

\subsection{Segmentation}

Image segmentation is one of the critical processes in the image processing. The segmentation process follows the region-based segmentation which checks the neighbouring seed points and if it gets matched with the initial seed point, it will be added to the region of segmentation. If it is not matched, the corresponding pixel will not be added to the region of segmentation. Another way of segmenting an image is cell segmentation. In this process, the image is converted into 2D image. Contrast can be adjusted for the better visualization of data to the algorithm developer. The data can be converted into binary data. Clean the data and overlay the perimeter on the given input image. A single image is partitioned into many segments, which turns into more meaningful and more straightforward to analyse [7]. This process leads to identify the specific objects and boundaries in the images. After segmentation, every pixel is labeled separately. If the same label is assigned to many pixels, it means that it shares specific characteristics. After labeling, all the segments will cover the full image.[8]

\subsection{Regions of Interest}

Among the available dataset, the subset of the sample is selected for a specific purpose. This process is called as Region of Interest (ROI). This concept is generally applied in the application of image processing. The process invthe olved in ROI detection is given in figure 3 . One of the best examples of ROI is medical imaging. To measure the size othe $f$ the tumor, ROI is used in an image. Another example is to access the cardiac function; the endocardial border is defined in a vision during different cardiac cycle stages like end-systole and end-diastole. For social purposes, ROI can be applied to the geographical information system by using the polygonal section in the 2D map.[9] In the computer vision application, ROI is used to define an object's borders, and in other implementations, the ROI can be used to ass symbolic labels to identify the content. ROI can be used to filter the image and it is implemented by utilizing a binary mask to the image. The binary image, which is the same size as the image in process with pixels, will be defined as a logical one and the remaining pixels as logical zero. 
P. Vijayakumar et al., International Journal of Emerging Trends in Engineering Research, 8(10), October 2020, 6750 - 6756

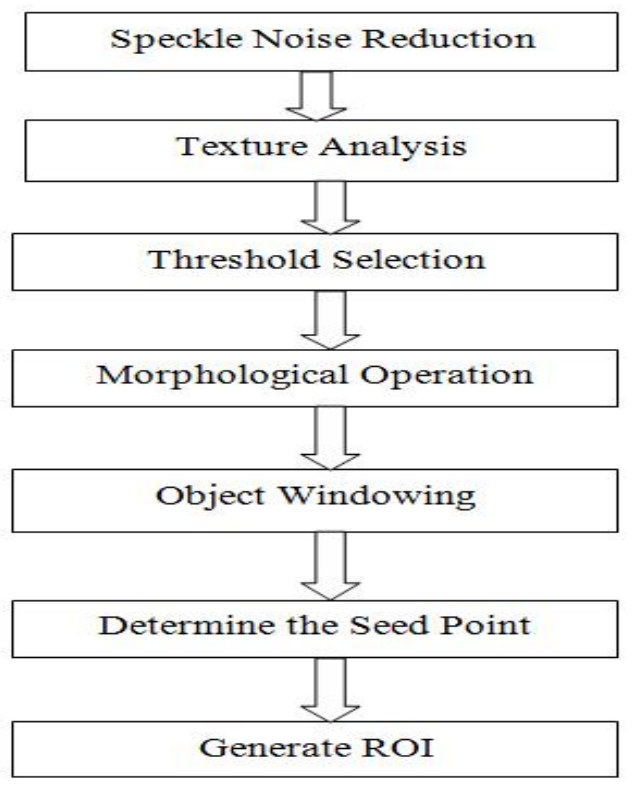

Figure 3: Steps involved in Region of Interest

\subsection{Texture Analysis}

Texture analysis is another critical process in image processing. It is used to characterize the region based on the texture content. This process is more useful when the objects in the image are characterized by texture more than the intensity or other threshold techniques. The texture analysis process is based on the values like range, the image's entropy, or standard deviation. From the defined values, if the information shows any variation in the intensity values of pixels. It leads to texture characterization. [10].

\subsection{Binary Images as a Mask}

The binary image as a mask is an essential step in ROI. Any binary image can be used for masking as the mask subjected to the binary image must be the same size as the image is filtered [2]. For example, the image to be filtered is the greyscale image I, the filtering can be done if the pixel values are more significant than 0.5 [11].

\subsection{Dilation and Erosion}

Dilation of a binary (0/1, o_/on) image expands contiguous regions of 'on' pixels in an image. It examines the neighboring pixels within a 'Window' around each pixel. If more than the specified fraction of neighboring pixels are 'on', then dilation turns the pixel 'on'. Pixels that are 'on' already are never altered. Erosion of a binary image [2] contracts contiguous regions of 'on' pixels in an image. It examines the neighboring pixels within a specified 'Window'. If more than the specified fraction of neighboring pixels are 'o_' then erosion turns the pixel 'o_'. Pixels that are 'o_' already are never altered.

\subsection{Pixel of Image}

In an image, a pixel is represented as a physical parameter which gives the addressable element. The address of the image depends on the physical coordinates. Each pixel value is taken from an original image to provide more accurate representations of the original.

\subsection{Seed Point Selection}

The selected regions will undergo in the ROI. The unselected areas will process through another selection step, which decides the correct seed point. The flow of seed pint selection is given in figure 4.The number of connected components needs to be determined to find the seed point. This process will identify the number of regions left in an image.[12] If only one area yet to get selected, then the unselected region will be chosen as the seed point.

\section{PARAMETER ANALYSIS}

The parametric analysis is used to find the abnormalities in a selected area. The defects are recorded in the histogram, which is compressed so that lower entries are improved.

In the histogram, the small peaks are representing the small area in the image are improved. The algorithm will detect a small area very accurately. Smoothening is applied to the histogram to avoid the insignificant local peaks in the complete area of an image. The smoothening technique is performed using the computational method. In this method, all the high-intensity pixel values are recalculated by calculating the average of a predefined number of neighboring pixels. [13]. Automatic detection of object by image analysis is the recent trend [14-16]. Automatic identification of objects using a mask width is defined experimentally and interactively by the user.

Higher width values are assigned to the larger area of the image, and lower width values are assigned to the image's smaller area. All fields in the picture are covered by assigning more upper and lower width values to the image. In the histogram, falling and rising edges are detected, so that number of valleys is detected. It will set up the threshold values. When the histogram starts to rise again, that point is named as the maximum local value. This value is used to segment objects and created a "label image." The peak value is more leads to the boundary moves to the canter of the kidney. 


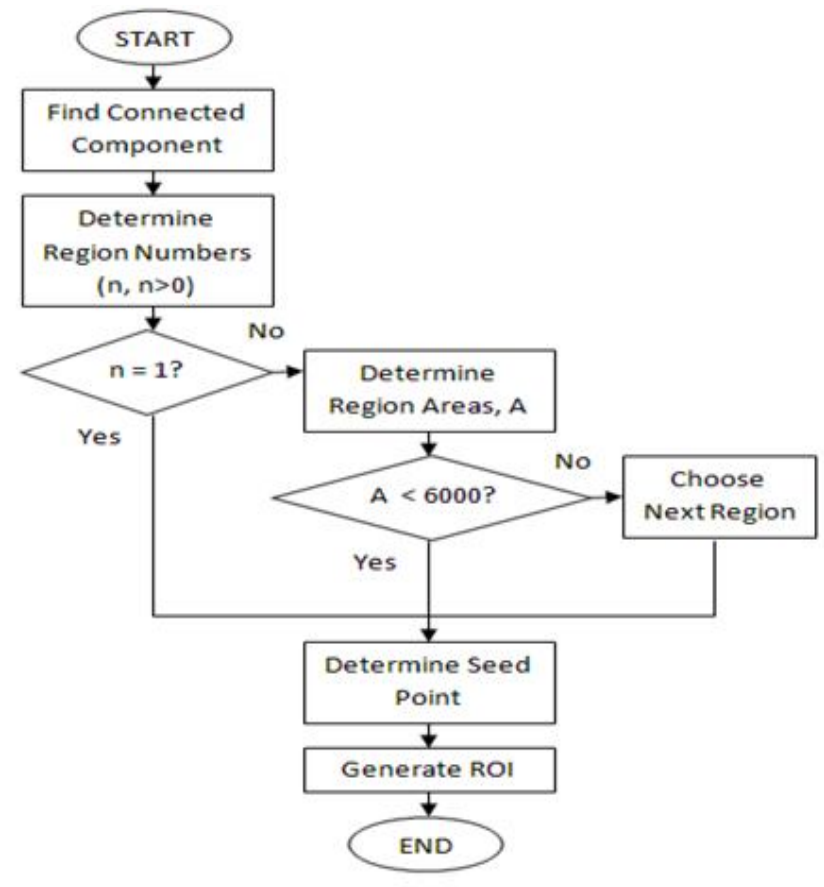

Figure 4: Flow chart for seed point selection

\section{SEGMENTATION ALGORITHMS}

The MRI dataset is created with the help of a segmentation framework. It is also used to train the systems, and the image noise can be removed by edge-preserving anisotropic diffusion. The segmentation algorithm has been modified to work on the region-orientation. It is used to recognize the organs accurately, and to get better results, the histogram values are normalized. The histogram values can be increased or decreased to identify more or less internal areas and area parameters to avoid select small objects in the recognition process. To recognize the abnormalities, the value of seed pixel and sensitivity is the most critical parameters. The segmentation algorithm is used to segment the kidney parts and their abnormalities. In this paper, two segmentation algorithms have been taken into consideration. They are region growing semiautomatic algorithm and feature extraction.

\subsection{Region Growing Semiautomatic Algorithm}

The essential operation of the semiautomatic algorithm is to find and demarcating organ structures. It will happen as a mass of neighboring pixels in an image. It will start seeing from the seed pixel. It is achieved by examining the intensity level of the adjacent pixels and begins from the seed pixel and spreads the select out the pixel based on grey level intensity values.[1] The intensity value is fixed based on the similarities between the adjacent pixels, and it must be in the same color to get belongs to the region. If the tolerance value is low, then the algorithm will select the reference pixel relatively equal. Simultaneously, a high tolerance value will allow the broader range of pixels to be included in the selected region, and it grows bigger. In practice, the organ image boundaries are taken and depending on the tolerance value chosen.[11] The image mask pixel represents the selected pixels' respective position in the original image to enhance efficiency. The proposed algorithm examines only the pixels that are not taken into consideration. The motive of the region growing method is to find the perfect definition of the boundary for the designated image.

\subsection{Feature Extraction}

Feature extraction is mainly used to reduce the dimensions of an image.[12] If the input data to the algorithm is extensive, and it is very tedious to process. So, it will be transformed into a set of features. This is called feature extraction.

\subsection{Threshold Value Selection}

The easiest way to segment the image by setting the threshold value. It is based on the clip level to turn an image into the binary image.

\section{RESULTS AND DISCUSSION}

\subsection{Normal Kidney}

Image segmentation has been used to find the boundaries or edges between the tissues to find a size measurement and by the image size, declare the abnormalities. Even though the ultrasound image is very easy and transferable and very protective but due to a large number of noises. It will affect the image

Figure 5 shows that the kidney is normal and Figure 6 shows parameters of the same. The value of seed pixel and the sensitivity are defined and used for the recognition of abnormalities [2].

\subsection{Abnormality Detection}

This method provides kidney abnormality, which presents inside the kidney by using MATLAB. This method is tested by using collected database MRI images. Figure 7 shows the abnormality of the kidney image by detecting the region of interest. Based on the threshold value, segmentation can be performed with the labeled image [2] [5]. 
P. Vijayakumar et al., International Journal of Emerging Trends in Engineering Research, 8(10), October 2020, 6750 - 6756

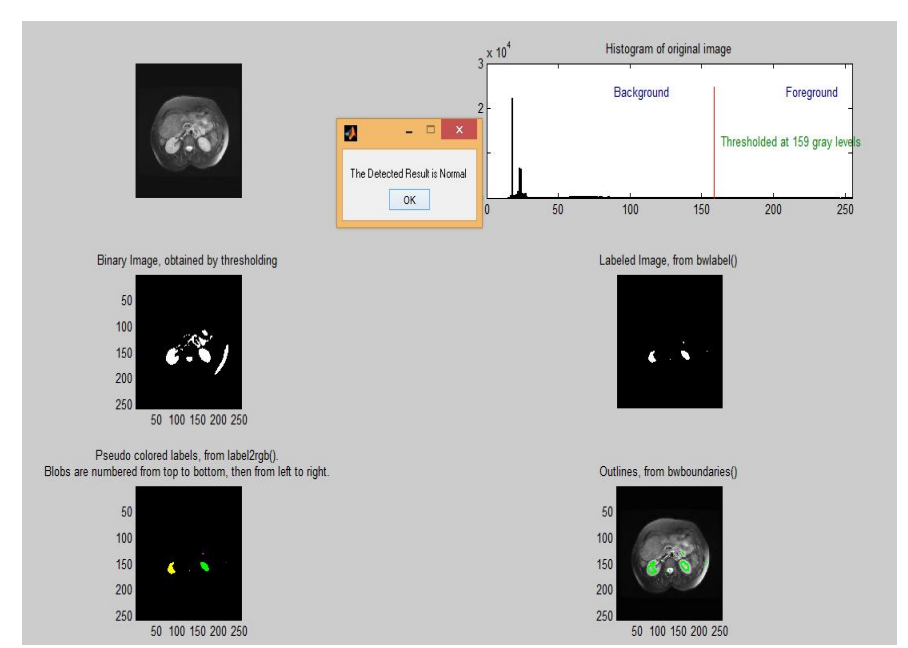

Figure 5: Normal kidney

\begin{tabular}{|c|c|c|c|c|c|c|}
\hline \multicolumn{7}{|c|}{ Command Window } \\
\hline \multicolumn{7}{|c|}{ (i) New to MATLAB? Watch this Video, see Eramples, or reed Getting Stated. } \\
\hline \multirow{2}{*}{$\begin{array}{l}\text { Segment } \# \\
\neq 1\end{array}$} & \multicolumn{2}{|c|}{ Mean Intensity Area } & \multicolumn{2}{|c|}{ Perimeter 0} & Centroid & \multirow{2}{*}{$\begin{array}{l}\text { Diameter } \\
17.4\end{array}$} \\
\hline & 185.6 & 239.0 & 74.4 & 86.0 & 158.4 & \\
\hline$\$ 2$ & 250.0 & 2.0 & 2.0 & 129.5 & 162.0 & 1.6 \\
\hline$\$ 3$ & 204.2 & 232.0 & 60.9 & 166.4 & 154.2 & 17.2 \\
\hline$\$ 4$ & 201.6 & 9.0 & 11.1 & 162.4 & 129.4 & 3.4 \\
\hline$\$ 5$ & 199.7 & 3.0 & 4.0 & 218.0 & 151.0 & 2.0 \\
\hline$\$ 6$ & 191.5 & 2.0 & 2.0 & 219.0 & 147.5 & 1.6 \\
\hline \multicolumn{7}{|c|}{ Finished running arun.m } \\
\hline \multicolumn{7}{|c|}{ Elapsed time $=17.70$ seconds. } \\
\hline \multicolumn{7}{|c|}{ Check out the figure window for the images. } \\
\hline \multicolumn{7}{|c|}{$\begin{array}{l}\text { Check out the cormand window for the numerica } \\
f x\rangle \gg\end{array}$} \\
\hline
\end{tabular}

Figure 6: Parameter Values of Normal kidney

\subsection{Stone Detection}

Figure. 8 shows the stone problem can be detected [4] by performing the original image get re-sized and then threshold value fixed to provide image clarity [2]. Based on that, segmentation can be done. Labeling and pseudo-random color provided for the identification of segmentation parts. According to the boundary detection and parameters of the image are defined with the greyscale value. Connectivity analysis is used to find the relation between neighborhood pixels [9]. Based on that, diagnosis can be identified. The parameters obtained for stone detection is mentioned in Figure 9.

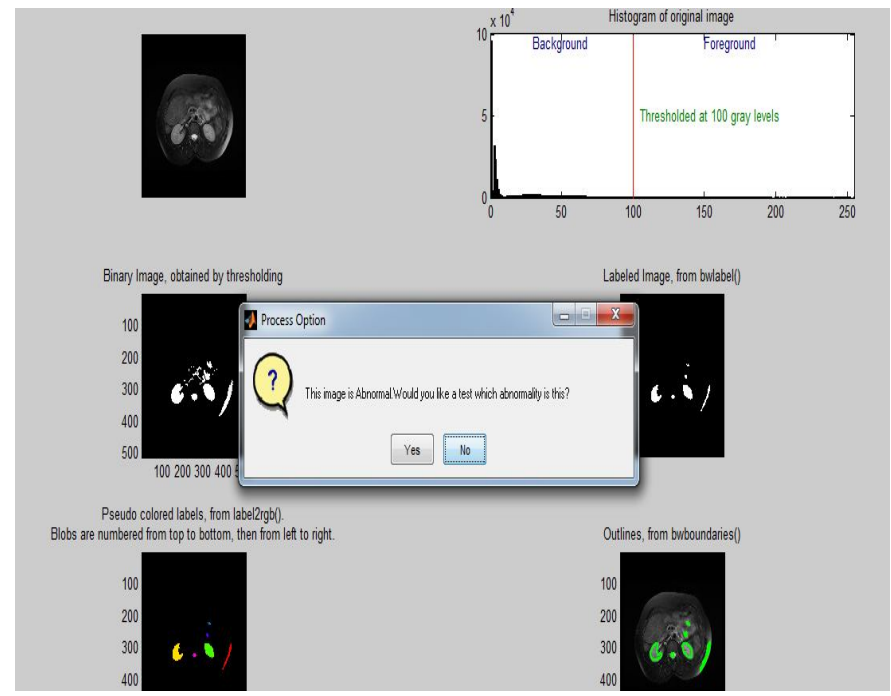

Figure 7: Abnormality detection

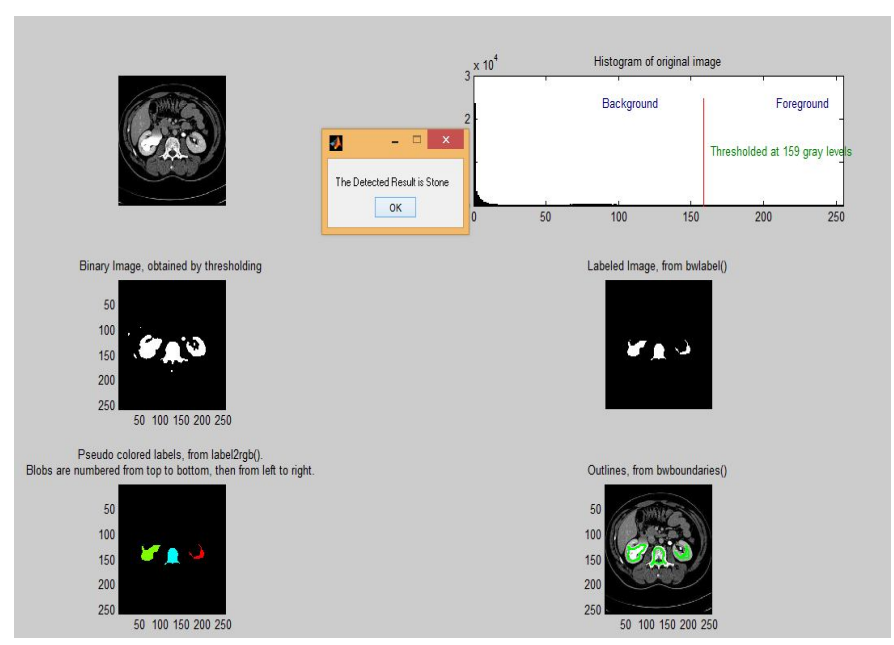

Figure 8: Stone detection

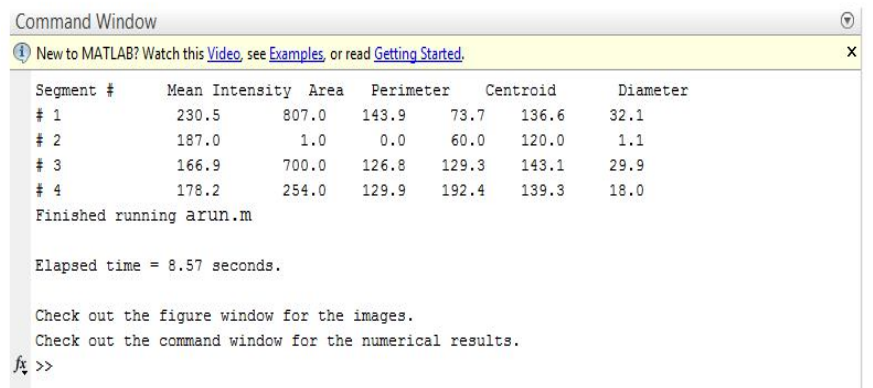

Figure 9: Parameter Values of Stone detection

\subsection{Cyst Detection}

Figure 10 shows the identification of the cyst problem present inside the kidney and its parameters are mentioned in Figure 11.[2] 


\subsection{Tumour Detection}

In order to obtain the tumor problem [5] present inside the kidney, a particular region can be identified with the corresponding parameters such as width, length [6]. For removing noise, the morphological operation is used. Pseudorandom color provided for identification of various segmentation parts shown in Figure 12 and the parameter values of tumor detection is mentioned in Figure 13.

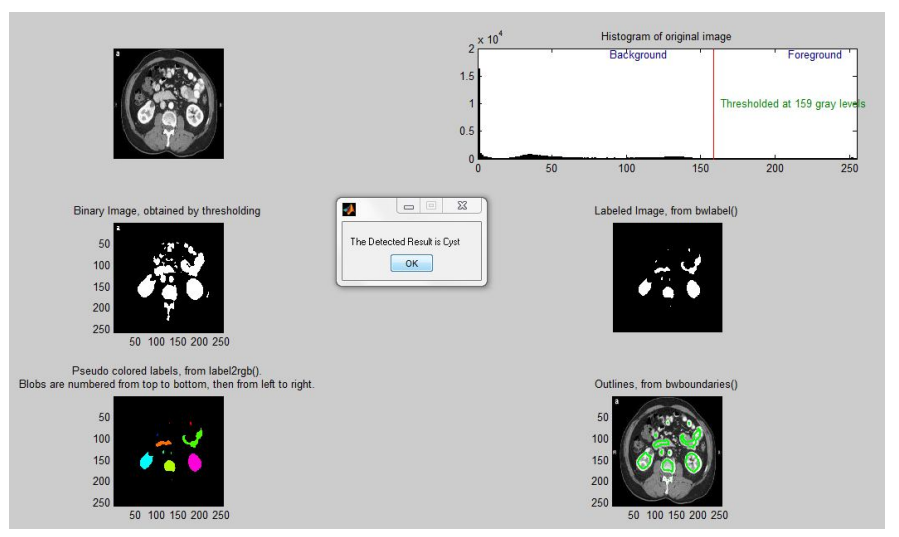

Figure 10: Cyst detection

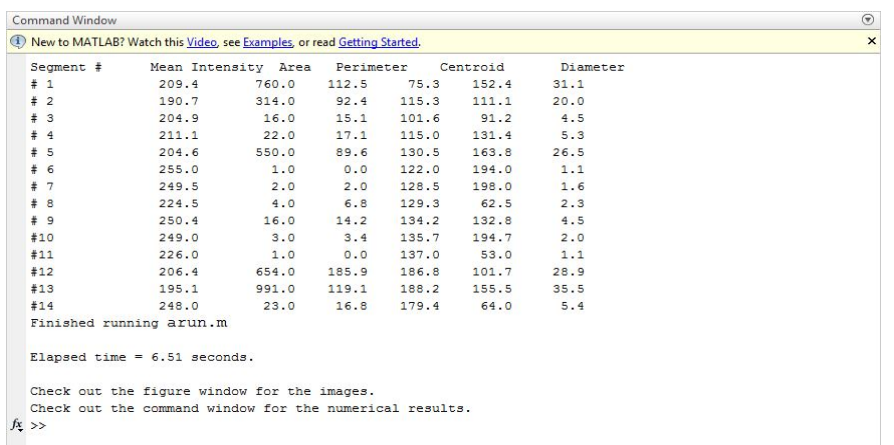

Figure 11: Parameter Values of Cyst detection

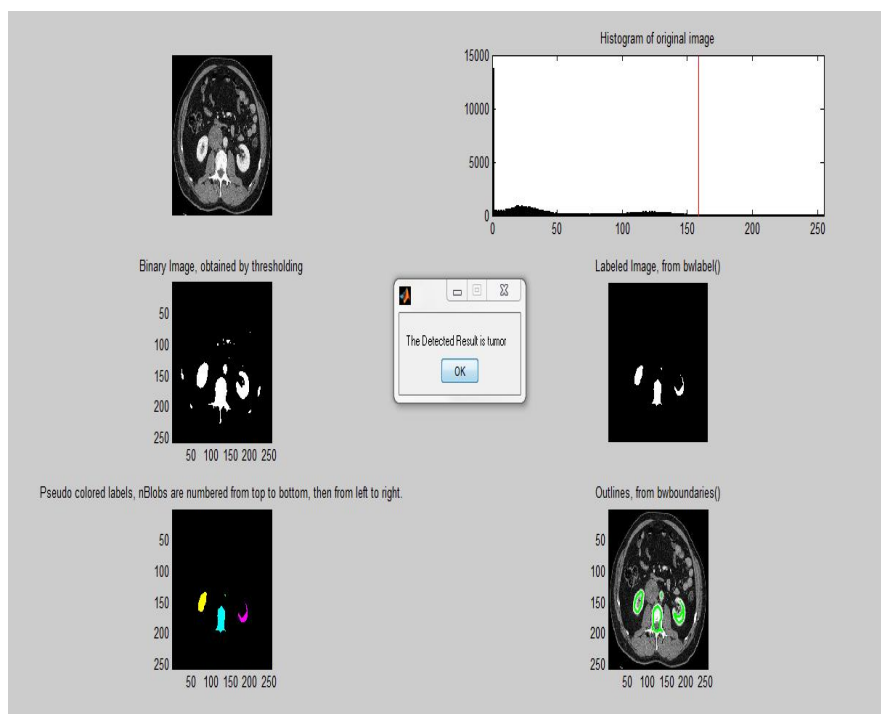

Figure 12: Tumour detection

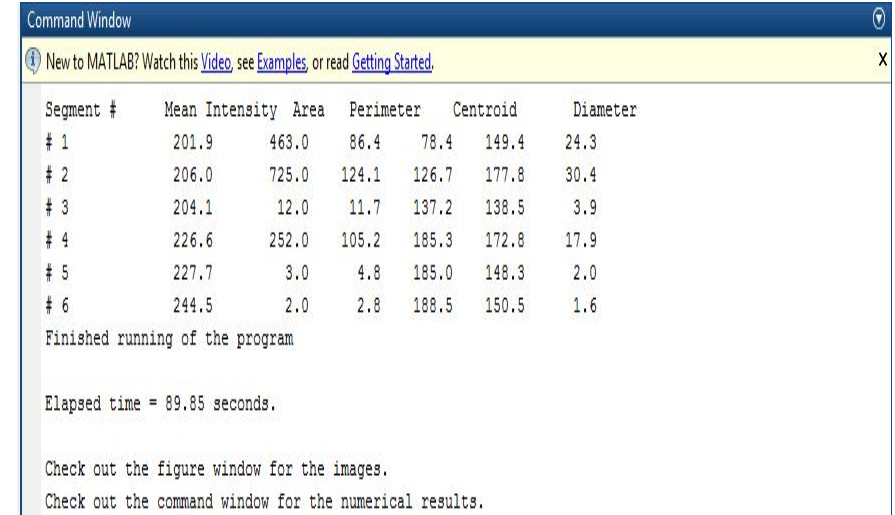

Figure 13: Tumour detection

\section{CONCLUSION}

The proposed method improves automation to find and remove areas of interest and have real values by specifying the actual size of the organ structure and any abnormalities in tissue regions. It gives the scope for a method of increasing automation to identify important areas based on the data in the database that are initially created. The system identifies organ structures based on their features and facilitates the detection of abnormalities. The proposed method provides good visualization for the region of interest due to the simplified ad high speed of image annotation process even in very difficult datasets. This enables $100 \%$ of accuracy in organ identification and $96 \%$ of accuracy in 30 abnormality identification from the input stage of the MRI image datasets. This work can be further extended to image communication by using a content-based image compression technique. By this technique, we can reduce the memory consumption of the image data base and also the memory compensation errors.

\section{REFERENCES}

1. D. T. Lin, C. C. Lei, and S. W. Hung, "Computer-aided kidney segmentation on abdominal CT images," IEEE Trans. Inform. Tech. Biomed., vol. 10, no. 1, pp. 59-65, Jan. 2006.

2. Skounakis E, Banitsas K, Badii A, Tzoulakis S, aravelakis E and Konstantaras A," ATD: A Multiplatform for Semiautomatic 3-D Detection of Kidneys and Their Pathology in Real Time," IEEE Trans. On Human-Machine Systems, vol. 44, no.1, pp. 146-153, Feb. 2014.

3. F. Khalifa, A. El-Baz, G. Gimel'farb, and M. Abu El-Ghar, "Non-invasive image- based approach for early detection of acute renal rejection," in Proc. Mid. Image Comput. Comput.-Assisted Intervention Conf. 2010, 2010, pp. 10-18. 
P. Vijayakumar et al., International Journal of Emerging Trends in Engineering Research, 8(10), October 2020, 6750 - 6756

4. P. R. Tamilselvi and P. Thangaraj, "Computer aided diagnosis system for stone detection and early detection of kidney stones," J. Comput. Sci., vol. 7, no. 2, pp. 250-254, 2011.

5. X. Chen, R. Summers, and J. Yao, "FEM-based 3-D tumor gowth prediction for kidney tumor," IEEE Trans. Bomed. Eng., vol. 58, no. 3, pp. 463-467, Mar. 2011

6. J. S. Jose, R. Sivakami, N. U. Maheswari, and R. Venkatesh, "An efficient diagnosis of kidney images using association rules," Int. J. Comput.Technol. Electron. Eng., vol. 12, no. 2, pp. 14-20, 2012.

7. R. Prevost, B. Mory, J. Correas, L. D. Cohen, and R. Ardon, "Kidney detection and real-time segmentation in 3D contrast-enhanced ultrasound images," in Proc. 9th IEEE Int. Symp. Biomed. Imag. ISBI, Barcelona, Spain, 2012, pp. 1559-1562.

8. F. Khalifa, M. A. Ghar, and T. Diasty, "Dynamic contrast-enhanced MRIbased early detection of acute renal transplant rejection," IEEE Trans. Med. Imag., vol. 32, no. 10, Oct. 2013.

9. P.Perona and J.Malik, "scale-space and edge detection using anisotropic discussion", IEEE Trans. Pattern Anal.Mach. Intell., vol.12, no.7,pp.629-639, Jul.1990.

10. H.Fujita and M.Fejri,"New trends in software methodologies, tools and techniques: How to create the magic wand?" in Proc.th soMeT-06,2006, pp.127-132.

11. Khudov, H., et al. "The Optimization Technique for Joint Discrete Search and Detection of Observation Objects." International Journal of Emerging Trends in Engineering Research 8 (2020): 2.

12. Nawaz, Nishad. "Artificial Intelligence Face Recognition for Applicant Tracking System." International Journal of Emerging Trends in Engineering Research 7 (2019): 12.

13. Gattim, Naveen Kishore, et al. "Plant Leaf Disease Detection Using SVM Technique." International Journal of Emerging Trends in Engineering Research 7.11 (2019): 634-637.

14. Vijayakumar Ponnusamy,abajieet,balaji,sangeetha,"A Palm Vein Recognition System based on support vector machine", IEIE Transaction on smart processing and computing, vol. 8, no. 1, February 2019.

15. Vijayakumar Ponnusamy, C. Amrith, S.S. Akhash, S. Sanoj and Shashank Srikan,Automatic Robotic Crop Disease Detection and Pesticide Dispenser Using Machine Learning,Journal of Advanced Research in Dynamical and Control Systems, Volume 11 | Issue 11, Pages: 119-125.

16. P.Vijayakumar, Abhishek.R, Kundala Sandeep,"Hybrid Classifier Based Content Based Image Retrieval", Indian journal of science and technology, Vol 9(46), December 2016, pp.1-7 